\title{
An Effective and Efficient FCM for Segmenting Color Image with High Intensity Noise \\ Weiling Cai, Li Wang
}

Department of Computer Science and Technology, Nanjing Normal University, Nanjing, China

Keywords: fuzzy c-means clustering; fast clustering; alpha-trimmed; image segmentation.

\begin{abstract}
In this paper, an effective and efficient fuzzy c-means algorithm is proposed for color image segmentation. To make the segmentation robust, the pixel similarity, the sliding window and alpha-trimmed mean are combined together to suppress the influence of heavy noise during the segmentation process. On the other hand, to make the segmentation effective, the quantitative technique is utilized in RGB space to accelerate the speed of segmentation. Our algorithm has two main advantages: (1) By considering the local spatial and color information together, the high intensity noise can be removed effectively to great extent; (2) Due to the using of quantitative techniques in RGB spaces, the time complexity of our algorithm is proportional to the number of colors (only 216) and independent of the number image pixels, and therefore it is very suitable for large-scale images. The experiments show that our algorithm is effective and efficient.
\end{abstract}

\section{Introduction}

Image segmentation is widely used in many fields, such as medical imaging, face recognition, robot vision, and so on. In general, images and video signals are frequently be corrupted by impulse noise in the process of image acquisition and transmission. The high intensity noise will bring more severe damage in image segmentation. Therefore, it's necessary to remove impulse noise before image segmentation.

Classically, image segmentation is defined as the partitioning of an image into non-overlapped, consistent regions which are homogeneous with respect to some characteristics such as gray value or texture [1]. Fuzzy c-means (FCM) uses fuzziness for the belongingness of each pixel to segmentation. It's more precise then hard methods. Recently, many researchers proposed efficient extensions of FCM methods for image segmentation. The techniques adopted in these methods can be divided into three groups: pre-processing technique [1-4], modified objective-function technique [5], post-processing technique [6]. The pre-processing technique (algorithm) aims to clean noise before image segmentation. The modified objective-function technique means that an anti-noise part is incorporated into the segmentation objective function. The post-processing technology removes the noise after image segmentation.

Recently, many researchers focus on the first kind of methods, and here we will review some of pre-processing algorithms. EnFCM [2] uses a linear weighted sum from the sliding window, and then performs the Fast FCM on the image. FGFCM defines a local similarity measure to reflect the relationship between pixels, and then segments the image using the Fast FCM algorithm. FCMDN [3] is the extension of FGFCM. After calculating the similarity, FCMDN only uses the dependable neighbors to decide the segmentation. NSWFCM [4] gets a linear weight sum image before its computation, and then uses spatially weighted FCM. These methods are briefness and easy to understand, and perform well with low intensity noise. But most of algorithms will fail with high intensity noise. Moreover, most of these algorithms only apply to gray images and does not suit to color images.

In order to overcome these shortcomings, we propose an effective and efficient fuzzy c-means algorithm for color image segmentation. The use of similarity measure, the sliding window method and alpha-trimmed mean can effectively remove high intensity noise. The time complexity is proportional to the number of colors after the using of quantitative techniques in RGB spaces. 
The rest of paper is organized as follows: In section 2, FCM, and Enhanced fuzzy c means clustering (EnFCM) algorithms are introduced. In section 3, we propose our algorithm. The experiments are presented in Section 4. Finally, conclusions and future work is given in Section 5.

\section{FCM}

In the FCM clustering algorithm, the objective function $\left(\mathrm{J}_{\mathrm{F}}\right)$ is as follow, the $\mathrm{U}$ and $\mathrm{V}$ are membership matrix and the cluster centers, respectively:

$$
J_{F}=\sum_{i=1}^{c} \sum_{j=1}^{N} u_{i j}^{m}\left(x_{j}-v_{i}\right)^{2}, \text { by } \sum_{i=1}^{c} u_{i j}=1 \quad \text { (1) where } \mathrm{X}=\left\{\mathrm{x}_{1}, \mathrm{x}_{2} \ldots \mathrm{x}_{\mathrm{n}}\right\} \text { is the gray }
$$

value of pixels, $\mathrm{N}$ is the number of the image pixels and $\mathrm{c}$ is the number of clusters. The value of $\mathrm{m}$ determines the fuzziness of the resulting classification.

EnFCM is a new extension of FCM algorithm. EnFCM uses a linearly weight sum on original image to generate a new image $\zeta$ :

$$
\xi_{k}=\frac{1}{1+\partial}\left(x_{k}+\frac{\partial}{N_{R}} \sum_{j \in N_{k}} x_{j}\right),
$$

(2) And then, to accelerate the

speed of algorithm, the fast FCM algorithm is used. Its objective function is $\mathrm{J}_{\text {Enfcm }}$ (3):

$$
J_{\text {Enfcm }}=\sum_{i=1}^{c} \sum_{l=1}^{q} n_{l} u_{i l}^{m}\left(\xi_{l}-v_{i}\right)^{2,} \text { by } \sum_{i=1}^{c} u_{i l}=1
$$

where $\mathrm{x}_{\mathrm{i}}, \mathrm{u}_{\mathrm{il}}$ are still the center of the ith cluster and the fuzzy membership of gray value $\mathrm{l}$ respect to cluster $\mathrm{i}$. $\mathrm{Q}$ is the gray level after quantitative techniques in gray space $(\mathrm{q}<<\mathrm{N}), \mathrm{n}_{1}$ is the number of pixels have the gray value $l\left(\mathrm{~N}\right.$ is equal to the sum of $\left.n_{l}(l=1 \ldots . . q)\right)$. Taking the first derivatives of $\mathrm{J}_{\text {Enfcm }}$ with respect to $\mathrm{u}_{\mathrm{il}}$ and $\mathrm{v}_{\mathrm{i}}$, and zeroing them respectively, two conditions at its local extrema will be obtained as follows:

$$
\begin{aligned}
& u_{i l}=\left(\xi_{l}-v_{i}\right)^{-2 / m-1} / \sum_{j=1}^{c}\left(\xi_{l}-v_{j}\right)^{-2 / m-1} \\
& v_{i}=\sum_{l=1}^{q} n_{l} u_{i l}^{m} \xi_{l} / \sum_{l=1}^{q} n_{l} u_{i l}^{m}
\end{aligned}
$$

\section{EnFCM Algorithm}

Step 1: Initial class number c and set $\varepsilon>0$.

Step 2: Use Eq. (2) to compute the new picture $\zeta$.

Step 3: Update $\mathrm{u}_{\mathrm{il}}$ by using Eq. (4).

Step 4: Update $\mathrm{v}_{\mathrm{i}}$ by using Eq. (5).

Repeat Step 3 and Step 4 until $\left|\mathrm{v}_{\text {new }}-\mathrm{v}_{\text {old }}\right|<\varepsilon$, and $\mathrm{v}$ are cluster prototypes.

\section{Our Algorithm}

\subsection{Description of Our Algorithm}

In this paper, an effective and efficient fuzzy c-means algorithm (EEFCM) is proposed for color image segmentation. Since images and video signals are frequently be corrupted by impulse noise in the process of image acquisition and transmission, in our paper, we utilize the following four parts to resist the high intensity noise: alpha trimmed mean filter, similarity measure, sliding window method, and quantitative technique. The four parts will be described in more detail in the next paragraphs.

We at first employ the well-known alpha trimmed mean filter [7] which is one kind of order statistics filter. By using the sort and statistics, this method has the excellent robustness to noise. The idea of the method is as follows: after trimming the bottom and top $\alpha / 2$ pixels of a local window, the mean of those remaining pixels $\mathrm{W}_{\mathrm{k}}$ is defined as follows;

$$
W_{k}= \begin{cases}\frac{1}{2 k-1} \sum_{i=h-k+1}^{h+k-1} s_{i} & \text { if } n \text { is odd }(\mathrm{n}=2 * \mathrm{~h}-1) \\ \frac{1}{2 k} \sum_{i=h-k+1}^{h+k} s_{i} & \text { if } n \text { is } \operatorname{even}(\mathrm{n}=2 * \mathrm{~h})\end{cases}
$$


where $n$ is the number of pixels in the local window, si is the sorted pixel value in the local window, the value of $\mathrm{k}$ is the half number of pixels after alpha trimmed. If $\mathrm{k}=1$, the method is as same as median, and if $\mathrm{k}=\mathrm{h}$ the method takes the form of mean.

Based on $\mathrm{W}_{\mathrm{k}}$, the similarity measure $\mathrm{f}(\mathrm{i}, \mathrm{j})$ is presented to reflect the similarity between the pixel $\mathrm{i}$ and the pixel $\mathrm{j}$. It is defined as a product of the spatial similarity $\mathrm{f}_{1}(\mathrm{i}, \mathrm{j})$ and the color value similarity $\mathrm{f}_{2}(\mathrm{i}, \mathrm{j}) \cdot f(\mathrm{i}, \mathrm{j})=f_{1}(\mathrm{i}, \mathrm{j}) \times f_{2}(\mathrm{i}, \mathrm{j})$

$$
\begin{aligned}
& f_{1}(\mathrm{i}, \mathrm{j})=\exp \left(\frac{-\max \left(\left|\mathrm{p}_{j}-\mathrm{p}_{i}\right|,\left|\mathrm{q}_{j}-\mathrm{q}_{i}\right|\right)}{\lambda_{s}}\right) \\
& f_{2}(\mathrm{i}, \mathrm{j})=\exp \left(\frac{-\left(\mathbf{w}_{\mathbf{k}}-\mathbf{x}_{\mathbf{j}}\right)^{2}}{\lambda_{g} \times \sigma^{2}}\right), \quad \sigma=\sqrt{\frac{\sum_{j \in N i}\left(\mathbf{x}_{\mathbf{j}}-\mathbf{w}_{\mathbf{k}}\right)^{2}}{N_{R}}}
\end{aligned}
$$

where pixel $\mathrm{i}$ is the center and $\mathrm{j}$ is its neighboring pixel in a local window. For more information, please refer to [1].

In our algorithm, the sliding window is used for removing the noise with the aid of both the alpha trimmed mean filter in Eq. (6) and similarity measure in Eq. (7-9). Here we use Eq. (10) to generate a new image $\xi_{\mathrm{i}}$ :

$$
\xi_{i}=\sum_{j \in N_{i}} f(\mathrm{i}, \mathrm{j}) x_{j} / \sum_{j \in N_{i}} f(\mathrm{i}, \mathrm{j})
$$

In order to accelerate the

speed of algorithm, the quantitative technique is being used here. In RGB color space, we use the 216 universal-color [8] to replace 256*256*256 (it is permutation and combination of the $\mathrm{R}$ component, the $\mathrm{G}$ component, the $\mathrm{B}$ component), and consequently its computational complexity is reduced from $\mathrm{O}\left(\mathrm{NcI}_{1}\right)$ to $\mathrm{O}\left(\mathrm{qcI}_{2}\right)$ (In RGB color space, $\mathrm{q}=216, \mathrm{~N}$ is number of color pixel, $\mathrm{q}<<\mathrm{N}$ ). Its objective function is $\mathrm{J}$ :

$$
\begin{aligned}
& J=\sum_{i=1}^{c} \sum_{l=1}^{q} n_{l} \mathbf{u}_{i l}^{m}\left(\xi_{l}-\mathbf{v}_{i}\right)^{2,} \text { by } \sum_{i=1}^{c} \mathbf{u}_{i l}=1 \\
& \mathbf{u}_{\mathbf{i l}}=\left(\xi_{\mathbf{l}}-\mathbf{v}_{\mathbf{i}}\right)^{-2 / m-1} / \sum_{j=1}^{c}\left(\xi_{l}-\mathbf{v}_{\mathbf{j}}\right)^{-2 / m-1} \\
& \mathbf{v}_{\mathbf{i}}=\sum_{l=1}^{q} n_{l} \mathbf{u}_{i l}^{m} \xi_{l} / \sum_{l=1}^{q} n_{l} \mathbf{u}_{i l}^{m}
\end{aligned}
$$

where $\mathrm{v}_{\mathrm{i}}, \mathrm{u}_{\mathrm{il}}$ is still the center of the ith cluster and the fuzzy membership of color value $\mathrm{l}$ respect to cluster i.

Now the new algorithm can expressed as bellow:

Step 1: Initial class number and set $\varepsilon>0$, set the value of $\mathrm{k}$ to determine how many pixels can we leave in a local window.

Step 2: If it's a gray Image, use Eq. (7) to compute f (i, j). If it's an RGB Image, compute (R-f(i, j), $\mathrm{G}-\mathrm{f}(\mathrm{i}, \mathrm{j}), \mathrm{B}-\mathrm{f}(\mathrm{i}, \mathrm{j})$ ) separately, and then, $f(i, j)$ is combined with them.

Step 3: Use Eq. (10) to generate a new image $\xi$.

Step 4: Update the membership matrix by using Eq. (12).

Step 5: Update the cluster centers by using Eq. (13).

Repeat Step 4 and Step 5 until $\left|\mathrm{v}_{\text {new }}-\mathrm{V}_{\text {old }}\right|<\varepsilon$, and $\mathrm{v}$ are vectors of cluster prototypes.

\subsection{Algorithm Analysis}

For improving comprehension of algorithm in this paper, in this section we will give a local window as Fig. 1(a) to illustrate how to find dependable pixels. The value of pixel in a smooth region tends to be close to each other, but if it is a noise point, it will not close to others. In the $3 \times 3$ local window, Fig. 1 (b) is corrupted image $(0,255$ are noise points). After the use of (2), we can find the value of $f(i, j)$ at the noisy pixels 255 is 0.09 , implying these pixels will play unimportant role in (5) and thus the influence of noise is effectively suppressed.

This algorithm has two important points: 
(1) Efficient method. The similarity measure, the sliding window method and alpha-trimmed mean can effectively remove high intensity Salt and Pepper, Gaussian, Speckle noise;

(2) Fast method. After the using of quantitative techniques in RGB spaces, its computational complexity is reduced from $\mathrm{O}\left(\mathrm{NcI}_{1}\right)$ to $\mathrm{O}\left(\mathrm{qcI}_{2}\right), \mathrm{c}, \mathrm{I}_{1}$ and $\mathrm{I}_{2}\left(<\mathrm{I}_{1}\right.$, generally) is respectively the number of clusters, iterations. $\mathrm{N}$ is the number of pixels. When it is a large-scale image, the computational time is still small.

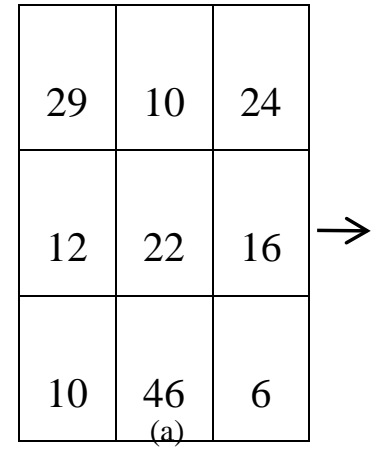

(b)

\begin{tabular}{|c|c|c|}
\hline 25 & 25 & 24 \\
5 & 5 & \\
\hline & & \\
12 & 0 & 16 \\
& & \\
\hline & & \\
10 & 0 & 0 \\
\hline
\end{tabular}$\quad$\begin{tabular}{ccc|c|}
\hline & 0.0 & 0.0 & 0.6 \\
86 & 86 & 56 \\
\hline & & \\
0.6 & 0.9 & 0.6 \\
59 & 95 & 59 \\
\hline & & \\
0.6 & 0.6 & 0.6 \\
59 & 56 & 56 \\
\hline
\end{tabular}

Fig.1: (a) The original image; (b) Corrupted image; (c) Value of $f(i, j)$;

\section{Experiments}

\subsection{Parameter Adjustment}

In our algorithm, we use the alpha-trimmed mean to remove high intensity Salt and Pepper, Gaussian, Speckle noise. Among them, the parameter $\mathrm{k}$ has enormous influence. We take parameter $\mathrm{k}$ change from 1 to 13 ( odd ) in a $5 \times 5$ window. As show in Fig 2, we can find that the value of the k parameter has great influence on Salt and pepper noise and Gauss noise. Specifically, along with the K increasing, the performance of the proposed algorithm is falling, if the type of noise is Salt and Pepper; when parameter $\mathrm{k}$ change from 1 to 13, if the type of noise is Gauss, the algorithm accuracy increases smoothly and then slowly falling; and for Speckle noise, parameter $k$ has little effect on the experimental results.

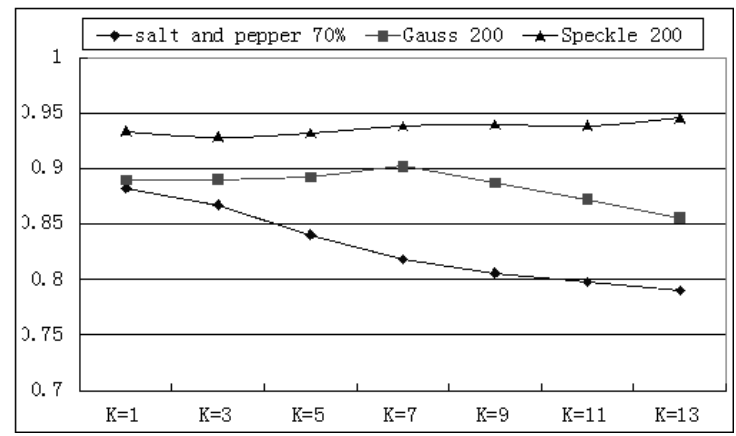

Fig.2: adjustment parameter $\mathrm{k}$

\subsection{Artificial Image}

Three types of noises are carried out in order to show the effective of our algorithm. At the same time, we will review some of pre-processing algorithms, like EnFCM, FGFCM. From the table 1, we can find that the correct rate of our algorithm is much higher than other algorithm. In addition, as show in Fig.4, our method has excellent visual performance. In particular, if we calculation $f(i, j)$ for two times, we found that our algorithm has higher accuracy for salt and pepper and Gauss noise, for Speckle noise, the experimental results change very little . 
Table 1: Results on artificial image

\begin{tabular}{|c|c|c|c|c|c|}
\hline Noise & $\begin{array}{l}\text { Fast } \\
\text { FCM }\end{array}$ & EnFCM & $\begin{array}{c}\text { FGFC } \\
\text { M }\end{array}$ & $\begin{array}{c}\text { EEFC } \\
M\end{array}$ & $\begin{array}{l}\text { EEFCM(twic } \\
\text { e) }\end{array}$ \\
\hline S\&P & \multirow{2}{*}{0.85353} & 0.9726 & 0.9969 & 0.9994 & \multirow{2}{*}{0.99977} \\
\hline $30 \%$ & & 2 & 6 & 3 & \\
\hline S\&P & \multirow{2}{*}{0.74623} & 0.9238 & 0.9812 & 0.9969 & \multirow{2}{*}{0.99736} \\
\hline $50 \%$ & & 9 & 3 & 6 & \\
\hline S\&P & \multirow{2}{*}{0.64995} & 0.7729 & 0.8894 & 0.9719 & \multirow{2}{*}{0.99426} \\
\hline $70 \%$ & & 4 & 6 & 9 & \\
\hline \multirow{2}{*}{$\mathrm{G} \sigma=100$} & \multirow{2}{*}{0.93434} & 0.9952 & 0.9981 & 0.9996 & \multirow{2}{*}{0.99977} \\
\hline & & 4 & 6 & 6 & \\
\hline $\mathrm{G} \sigma=150$ & 0.85146 & 0.9583 & 0.9920 & 0.9978 & \multirow{2}{*}{0.99805} \\
\hline \multirow[b]{2}{*}{$\mathrm{G} \sigma=200$} & \multirow[b]{2}{*}{0.77484} & $\begin{array}{c}3 \\
0901\end{array}$ & $\begin{array}{c}8 \\
0909\end{array}$ & $\begin{array}{c}8 \\
09958\end{array}$ & \\
\hline & & $\begin{array}{c}0.9001 \\
6\end{array}$ & $\begin{array}{c}0.9 / 98 \\
5\end{array}$ & $\begin{array}{c}0.9958 \\
7\end{array}$ & 0.99782 \\
\hline \multirow[t]{2}{*}{$\mathrm{S} \sigma=100$} & \multirow[t]{2}{*}{0.98008} & 0.9997 & 0.9979 & 0.9983 & \multirow[t]{2}{*}{0.99799} \\
\hline & & 7 & 9 & 9 & \\
\hline $\mathrm{S} \sigma=150$ & 0.92183 & 0.9863 & 0.9943 & 0.9953 & 0.99535 \\
\hline \multirow[b]{2}{*}{$\mathrm{S} \sigma=200$} & \multirow[b]{2}{*}{0.87385} & 0.9637 & 0.9888 & 0.9923 & \multirow[b]{2}{*}{0.99242} \\
\hline & & 3 & 7 & 7 & \\
\hline
\end{tabular}
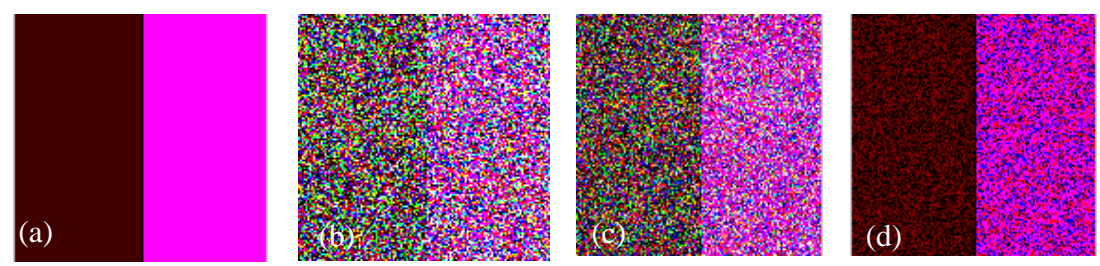

Fig.3: (a) Original image; (b) Salt and pepper 70\%; (c) Gauss $\sigma=200$; (d) Speckle $\sigma=200$;
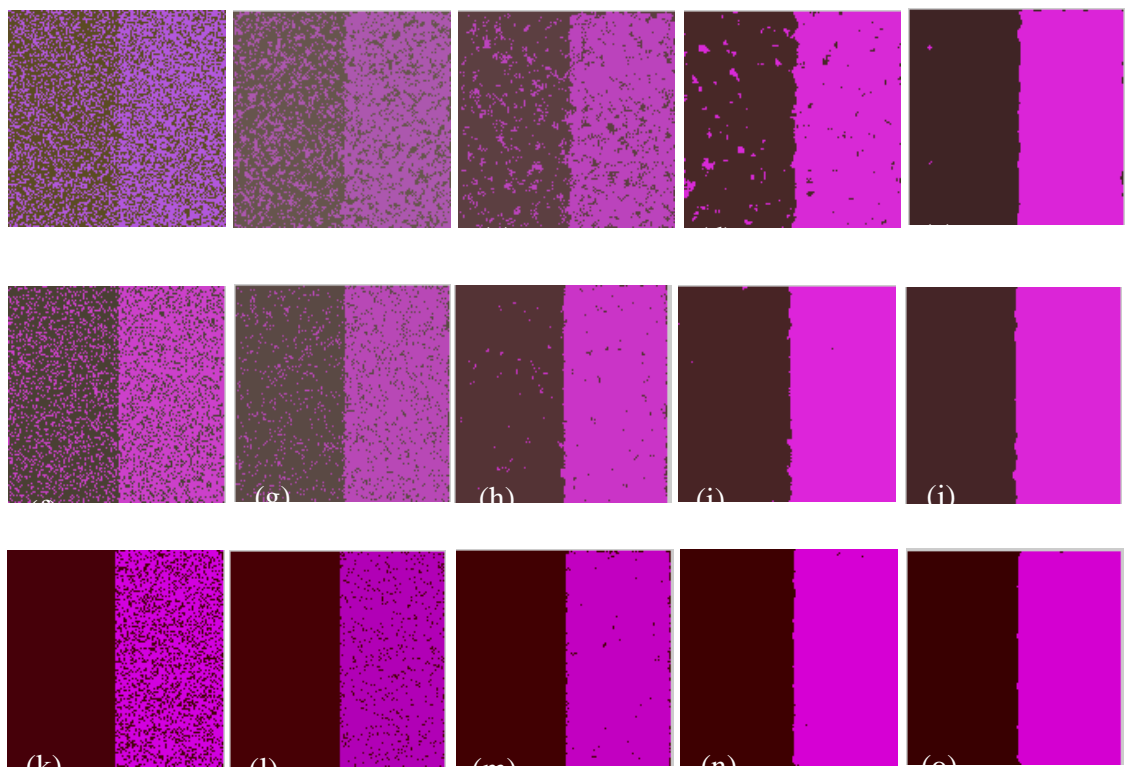

Fig.4: Segmentation results:(a-e)Fast FCM, EnFCM, FGFCM, EEFCM and EEFCM, twice on the image with Salt and pepper noise 70\%; (f-j) five algorithms on the image with Gauss noise $\sigma=200$ : (k-o) five algorithms on the image with Speckle $\sigma=200$;

\subsection{Real Image}

In this section, we compare our method with three approaches for removed impulse noise. To show the characteristics and performances of our algorithm, a variety of noises are carried out on real Image. From the table 2, the result shows that our algorithm has higher accuracy rate than other algorithm. Moreover, comparing with other methods, our algorithm has excellent psycho visual 
performance as shown in Fig.6. In particular, if the types of noise is Salt and pepper or Gauss, it can improve the performance of segmentation when we calculate $f(i, j)$ for two times.

Table 2: Results on real image

\begin{tabular}{lccccc}
\hline Noise & Fast FCM & EnFCM & FGFCM & EEFCM & EEFCM(twice) \\
\hline S\&P 30\% & 0.76240 & 0.85877 & 0.96268 & 0.97552 & $\mathbf{0 . 9 7 8 4 3}$ \\
S\&P 50\% & 0.62854 & 0.74240 & 0.87995 & 0.96402 & $\mathbf{0 . 9 6 4 7 2}$ \\
S\&P 70\% & 0.55316 & 0.64236 & 0.71421 & 0.88830 & $\mathbf{0 . 9 4 0 1 6}$ \\
G $\sigma=100$ & 0.76498 & 0.86851 & 0.94897 & 0.96325 & $\mathbf{0 . 9 6 4 8 7}$ \\
G $\sigma=150$ & 0.67496 & 0.75604 & 0.86217 & 0.94328 & $\mathbf{0 . 9 5 2 9 4}$ \\
G $\sigma=200$ & 0.62403 & 0.67666 & 0.78444 & 0.90768 & $\mathbf{0 . 9 3 4 0 0}$ \\
S $\sigma=100$ & 0.82248 & 0.96047 & 0.96008 & $\mathbf{0 . 9 6 5 6 7}$ & 0.96182 \\
S $\sigma=150$ & 0.74243 & 0.90745 & 0.93451 & $\mathbf{0 . 9 5 1 1 9}$ & 0.94554 \\
S $\sigma=200$ & 0.70091 & 0.83761 & 0.90150 & $\mathbf{0 . 9 4 5 3 1}$ & 0.94157 \\
\hline
\end{tabular}
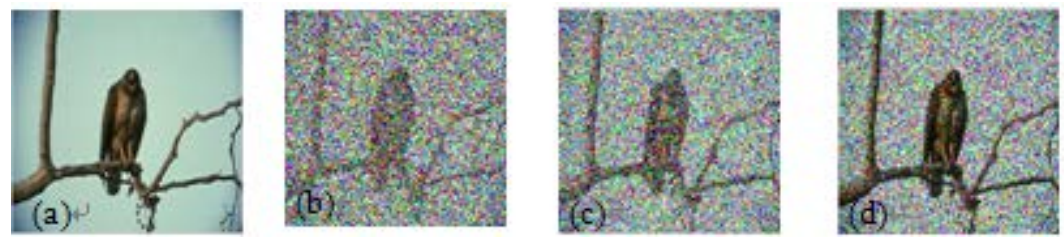

Fig.5: (a) Original image; (b) Salt and pepper 70\%; (c) Gauss $\sigma=200$; (d) Speckle $\sigma=200$;
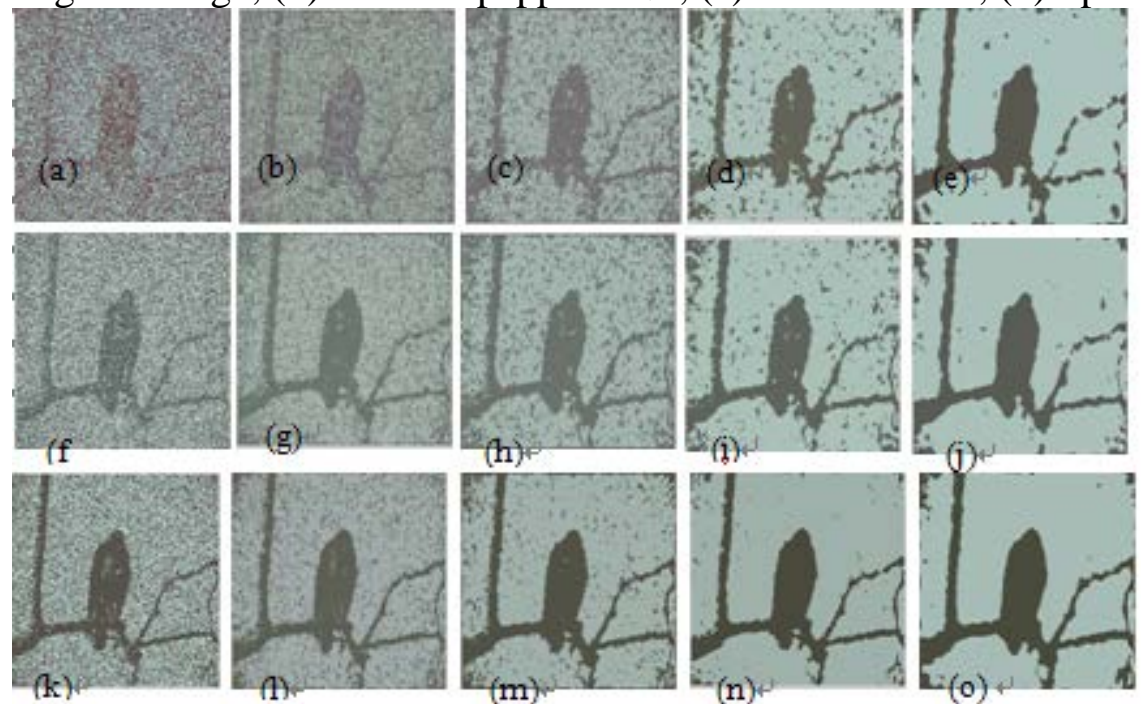

Fig.6: Segmentation results:(a-e) Fast FCM, EnFCM, FGFCM, EEFCM and EEFCM, twice on the image with Salt and pepper noise 70\%; (f-j) five algorithms on the image with Gauss noise $\sigma=200$ : (k-o) five algorithms on the image with Speckle $\sigma=200$;

\section{Conclusions}

In the proposed algorithm, the different type of high intensity noise, such as Salt and Pepper, Gaussian, Speckle noise, will be effectively removed. Meanwhile, this algorithm applies to gray image and color image.

\section{References}

[1] W. L. Cai, S. C. Chen, D. Q. Zhang. Fast and robust fuzzy c-means clustering algorithms incorporating local information for image segmentation. Pattern Recognition, 40(3), pp.825-838, 2007.

[2] L. Szilágyi, et. al. MR Brain image segmentation using an enhanced Fuzzy C-Means algorithm. IEEE EMBS, pp. 724-726, 2003. 
[3] W. L. Cai, S. C. Chen, L. Lei. A fuzzy clustering algorithm for image segmentation using dependable neighbor pixels, CJK Joint Workshop on Pattern Recognition (CJKPR), 840-844, 2009.

[4] S. Lu. L. Peng. Color image segmentation based on improved FCM algorithm incorporating spatial information. CISP2010, pp.1115-1118, 2010.

[5] D. Y. Tang, J. Yang, Y. S. Huang. Double weighed FCM algorithm for color image segmentation. Conference on Machine Learning and Cybernetics, Xian, July. 15-17, pp. 1135-1138, 2012.

[6] J. H. Xue, et.al. An integrated method of adaptive enhancement for unsupervised segmentation of MRI brain images, Pattern Recognition. Letters. vol. 24, pp. 2549-2560, 2003.

[7] F. Ahmed, S. Das, Removal of high density salt and pepper noise in images with an iterative adaptive fuzzy filter using alpha-trimmed mean, IEEE Trans. on Fuzzy Systems, 2013.

[8] R. C. Gonzalez, R. E. Woods. Digital Image Processing, Third Edition. pp. 426-428, 2005. 